\title{
Polarization-domain-wall complexes in fiber lasers
}

\author{
Caroline Lecaplain, ${ }^{1, *}$ Philippe Grelu, ${ }^{1}$ and Stefan Wabnitz ${ }^{2}$ \\ ${ }^{1}$ Laboratoire Interdisciplinaire Carnot de Bourgogne, U.M.R. 6303 C.N.R.S., Université de Bourgogne, 9 avenue A. Savary, \\ BP 47870 Dijon Cedex 21078, France \\ ${ }^{2}$ Dipartimento di Ingegneria dell'Informazione, Università di Brescia, Via Branze 38, Brescia 25123, Italy \\ *Corresponding author: Caroline.Lecaplain@u-bourgogne.fr
}

Received November 2, 2012; accepted November 20, 2012;

posted November 28, 2012 (Doc. ID 179008); published December 19, 2012

\begin{abstract}
We present a simple theoretical model that explains polarization switching in fiber ring lasers operating with a normal path-averaged dispersion and a typical intermediate level of birefringence. Such polarization dynamics, based on a type of polarization-domain-wall (PDW) structures, agree qualitatively well with our experimental observations. We also stress the complex and chaotic nature of the observed polarization-switching states. This is corroborated by detailed numerical simulations that predict the buildup of consecutive and transient PDW structures at the subnanosecond scale, which are not fully resolved experimentally. (c) 2012 Optical Society of America
\end{abstract}

OCIS codes: $\quad 190.5530,140.3510,190.4370$

\section{INTRODUCTION}

Mode-locked fiber lasers, besides being attractive sources of ultrashort optical pulses for numerous applications, constitute an ideal platform for the fundamental exploration of complex nonlinear dynamics. Pulse generation is initiated by the existence of a fast saturable absorber, while the stationary pulse results from a composite balance involving several dissipative and dispersive effects, better understood in the frame of the theory of dissipative solitons [1]. However, if the experimentalist happens to remove the fast saturable absorber element, the fiber laser cavity can still manifest pulsations at the cavity round-trip frequency, although much weaker and surrounded by an important noise level [2]. In the case of a fiber ring cavity, with a low level of polarization-dependent losses, the dominant feature of the observed pulsations consists in polarization-switching states [2-7]. The interpretation of these antiphase polarization dynamics is still controversial, as no simple physical model has been developed to date. Williams et al. [2] developed a model based on the gain dynamics that included cavity boundary conditions. Such a model successfully explained the buildup of polarization dynamics at the cavity round-trip frequency. However, the need for a simpler picture of the polarization-switching mechanism remained. In that spirit, the analogy of the polarization-switching dynamics with the formation of polarization-domain-wall (PDW) solitary waves was recently suggested [ $\underline{5}-\mathbf{7}]$. PDWs are localized nonlinear structures separating adjacent domains of different polarization eigenstates of a dispersive Kerr medium. They were predicted in 1994 [ㅇ,9] and observed a few years later in short lengths of optical fibers that featured normal dispersion and ultralow birefringence (spun fibers) [10]. Thus, the possible emergence of PDWs in fiber laser cavities, where the typical birefringence level is about two orders of magnitude higher than in spun fibers, requires a specific study. This study, which is developed in Section 2, constitutes the main contribution of the present article. In Section 3 , we present the results of our experimental observations, performed in an erbium-doped fiber (EDF) ring laser. In comparison to other recent experimental observations [5-7], we here point out the composite nature of the observed domain walls, and the multifaceted range of dynamics available while exploring the system parameters. This has motivated us to undertake detailed numerical simulations as well, which are presented in Section 4 and reveal the appearance of PDW complexes and transients at the subnanosecond scale.

\section{THEORY OF POLARIZATION-DOMAIN WALLS IN FIBER LASERS}

A typical fiber laser cavity operating at $1.5 \mu \mathrm{m}$, the wavelength of choice for optical communications, is made by combining and splicing several telecom fibers, such as standard singlemode fiber (SMF), EDF, dispersion-shifted fiber (DSF), and/ or dispersion-compensation fiber (DCF), all of which are usually nonpolarization-maintaining fibers. To mode lock such a fiber laser, the most popular and versatile technique consists in inserting a polarizer into the cavity: nonlinear polarization evolution that takes place during propagation in the fibers is thus followed by intensity discrimination when light passes through the polarizer, providing the overall effect of an ultrafast saturable absorber. Ultrashort pulses of high peak intensity may be generated; they are of scalar nature, since the polarization is constrained by the polarizer.

If we now remove the polarizing element, the light field recovers its vector nature. As the ultrafast saturable absorber effect has disappeared, we can rule out high-peak-intensity pulses. Instead, a quasi-continuous wave can be expected, whose average power will saturate the gain of the active fiber until it balances the total cavity losses. However, the existence of a strong noise background may trigger vector-wave dynamics, where the Kerr nonlinearity can be involved. After a few round trips, the dynamics is basically ruled by the interplay between the path-averaged fiber group velocity dispersion (GVD) and the Kerr nonlinearity. Indeed, for nearFourier-transform-limited pulses of picosecond durations and a cavity length of the order of $10 \mathrm{~m}$, the dispersion length 
$L_{d}$ in any fiber portion of the cavity remains significantly larger than the cavity length.

The bandwidth limitation of the gain may typically be neglected whenever the temporal structure of the intracavity field is in the range of picosecond to subnanosecond durations. Clearly, the transient effects associated with the gain dynamics can be neglected in the formation of subnanosecond vector pulse structures in class-B lasers such as EDF lasers; they would only occur over more than $10^{4}$ round trips, for a laser cavity length of the order of $10 \mathrm{~m}$.

We may thus consider, as a minimal model to represent and interpret the formation of relatively fast (i.e., in the picosecond range) wave dynamics in a polarizer-free fiber ring laser, the purely conservative interaction among the two polarization components of the fiber under the action of the GVD and the Kerr effect. Considering moderate intracavity pulse intensities at the Watt level-in contrast to mode-locked pulses that can attain the kilowatt level-nonlinear effects occur over a length scale $L_{\mathrm{nl}}$ that is in the range of $10^{2}-10^{3} \mathrm{~m}$. Therefore, nonlinear effects occur over a length scale that is much longer than the fiber birefringence beat length $L_{b}$, which is typically $1-$ $10 \mathrm{~m}$. The consequence is that nonlinear energy transfer among the two principal axes of the fiber can be neglected, since such effect is represented by rapidly oscillating terms that average to zero over the distance $L_{\mathrm{nl}}$ [11]. On the other hand, a cavity length $L$ of a few meters is much shorter than the correlation length $L_{c}$ (typically $50-100 \mathrm{~m}$ ) of the random birefringence fluctuations. Thus, random polarization-mode dispersion effects can be neglected and the propagation may be described in terms of a perfectly deterministic model. Therefore the propagation of the two polarization components of the electric field in a single-mode birefringent fiber is ruled by the following incoherently coupled nonlinear Schrödinger equations:

$$
\begin{aligned}
& i \frac{\partial A_{x}}{\partial Z}+i \delta \frac{\partial A_{x}}{\partial T}-\frac{\beta_{2}}{2} \frac{\partial^{2} A_{x}}{\partial T^{2}}+\gamma\left(\left|A_{x}\right|^{2}+\frac{2}{3}\left|A_{y}\right|^{2}\right) A_{x}=0, \\
& i \frac{\partial A_{y}}{\partial Z}-i \delta \frac{\partial A_{y}}{\partial T}-\frac{\beta_{2}}{2} \frac{\partial^{2} A_{y}}{\partial T^{2}}+\gamma\left(\left|A_{y}\right|^{2}+\frac{2}{3}\left|A_{x}\right|^{2}\right) A_{y}=0,
\end{aligned}
$$

where $E_{x, y}=A_{x, y} \exp \left[i \beta_{x, y} Z\right]$ are the complex envelopes of the field in the $x$ or $y$ polarization with propagation constant $\beta_{x}$ and $\beta_{y}$, respectively, $\delta=\delta n / 2 c, \delta n=n_{x}-n_{y}$ is the refractiveindex difference between the two polarizations, $\beta_{2}$ is the GVD coefficient (which is supposed to be equal for the two polarizations), $\gamma$ is the nonlinear coefficient, $Z$ is the distance, and $T$ is a retarded time in a reference frame traveling with the average group velocity (GV) of the two polarizations. Equation (1) can be simplified to

$$
\begin{aligned}
& i \frac{\partial a_{x}}{\partial Z}-\frac{\beta_{2}}{2} \frac{\partial^{2} a_{x}}{\partial T^{2}}+\gamma\left(\left|a_{x}\right|^{2}+\frac{2}{3}\left|a_{y}\right|^{2}\right) a_{x}=0, \\
& i \frac{\partial a_{y}}{\partial Z}-\frac{\beta_{2}}{2} \frac{\partial^{2} a_{y}}{\partial T^{2}}+\gamma\left(\left|a_{y}\right|^{2}+\frac{2}{3}\left|a_{x}\right|^{2}\right) a_{y}=0,
\end{aligned}
$$

where $a_{x, y}=A_{x, y} \exp \left[ \pm i \Omega T+i \beta_{2} \Omega^{2} Z / 2\right]$ are the new complex amplitudes, which are frequency shifted by $\pm \Omega$ (where $\Omega=\delta / \beta_{2}$ ), respectively. Following [요, $\underline{-}$ ], let us consider the solitary-wave solutions of Eq. (2) of the form $a_{x, y}(Z, T)=e_{x, y}(T) \exp \left[i k_{x, y} Z\right]$, so that one obtains the two coupled ordinary differential equations (ODEs)

$$
\begin{gathered}
\frac{\beta_{2}}{2} \frac{d^{2} e_{x}}{d T^{2}}=-k_{x} e_{x}+\gamma e_{x}^{3}+\frac{2}{3} \gamma e_{x} e_{y}^{2}, \\
\frac{\beta_{2}}{2} \frac{d^{2} e_{y}}{d T^{2}}=-k_{y} e_{y}+\gamma e_{y}^{3}+\frac{2}{3} \gamma e_{y} e_{x}^{2} .
\end{gathered}
$$

The above equations can be simplified, in the normal dispersion regime $\left(\beta_{2}>0\right)$, by defining the new variables $u=e_{x}\left(\gamma / k_{x}\right)^{1 / 2}, v=e_{y}\left(\gamma / k_{x}\right)^{1 / 2}, t=T\left(k_{x} / \beta_{2}\right)^{1 / 2}$, which leads to the two coupled, second-order ODEs:

$$
\begin{aligned}
& \ddot{u}=-2 u+2 u^{3}+\frac{4}{3} u v^{2}, \\
& \ddot{v}=-2 \beta v+2 v^{3}+\frac{4}{3} v u^{2} .
\end{aligned}
$$

Here, the overdots stand for derivatives with respect to the variable $t$, and $\beta \equiv k_{y} / k_{x}$. Equation (4) describes the motion of a particle in the bidimensional potential $V(u, v)=u^{2}+$ $\beta v^{2}-u^{4} / 2-v^{4} / 2-2 u^{2} v^{2} / 3$. As shown in Fig. 1, the potential $V$ has a local minimum at the origin $\left(u_{m}, v_{m}\right)=(0,0)$. Moreover, Fig. 1 also shows that, whenever $\beta$ grows larger than the bifurcation value $\beta_{\text {bif }}=2 / 3$, the two equal maxima at $u= \pm 1$ and $v=0$ split into four equal maxima in each quadrant. For example, Fig. 1(b) shows that for $\beta=1$ the four maxima are symmetrically located at $\left(\left|u_{M, i}\right|,\left|v_{M, i}\right|\right)=(\sqrt{3 / 5}, \sqrt{3 / 5})$, with $i=1,2,3$, 4. Equation (4) has special solutions corresponding to separatrix trajectories connecting different maxima of the potential $V$. The separatrices connecting maxima in opposite quadrants correspond to vector dark solitons, whereas the separatrices connecting maxima in adjacent quadrants correspond to the so-called PDW solitary waves $[\underline{8}, 9]$.

The PDW represents a time-localized transition between two semi-infinite domains of uniform polarization; its behavior is illustrated in Fig. 2, which has been obtained from Eq. (4) by a standard shooting numerical integration procedure. In the shooting method, we consider a finite temporal window, say, $\left(t_{-}, t_{+}\right)$, and we fix as the boundary conditions the values $\left(u\left(t_{-}\right), u\left(t_{+}\right)\right)$and $\left(v\left(t_{-}\right), v\left(t_{+}\right)\right)$, which are located on different maxima of $V$. As an initial guess for the solution, we simply suppose that $u$ and $v$ remain equal to one of the maxima $\left(u_{M, i}, v_{M, i}\right)$ at all times. The resulting evolution for the field amplitudes $u(t)$ and $v(t)$, as shown in Fig. 2, corresponds to the separatrix emanating from $\left(u_{M, 3}, v_{M, 3}\right)$ or the maximum of $V$ in the bottom left (or third) quadrant of Fig. 1(b), and reaching the maximum $\left(u_{M, 2}, v_{M, 2}\right)$ in the upper left (or second) quadrant. The initial condition of the shooting procedure here is $\left(u_{\text {in }}(t), v_{\text {in }}(t)\right)=\left(u_{M, 3}, v_{M, 3}\right)$. As can be seen, the $u$ envelope switches from negative to positive values,
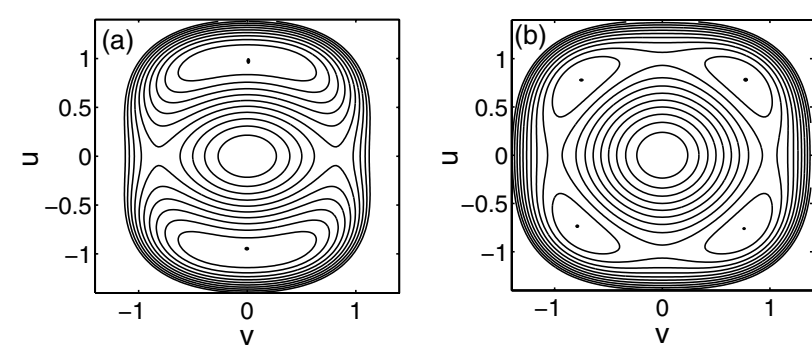

Fig. 1. Contour plot of the potential function $V(u, v)$ for a birefringent fiber; black dots indicate its maxima. (a) Case with $\beta=2 / 3$ and (b) case with $\beta=1$. 

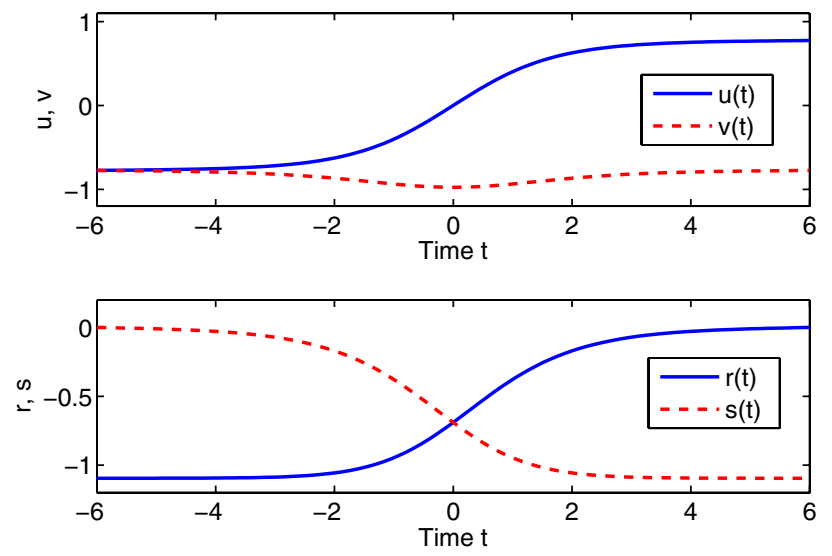

Fig. 2. (Color online) Evolution with time $t$ of the $(u, v)$ or $(r, s)$ components of a polarization-domain wall in a birefringent fiber.

whereas the $v$ envelope only exhibits a small hump in the correspondence of the transition of the $u$ envelope. In Fig. 2 , we also show the evolution of the polarization components $r(t)=$ $(u(t)+v(t)) / \sqrt{2}$ and $s(t)=(u(t)-v(t)) / \sqrt{2}$ that correspond to a reference frame rotated at 45 degrees with respect to the principal axes of the fiber. This clearly shows that the domain wall represents a full switching among orthogonal states of polarization. Note that, since Eqs. (3) are invariant if we introduce in the solitary-wave solution ansatz an arbitrary (in particular, equal to $\pi / 2$ ) constant phase shift among the two polarization components $a_{x}$ and $a_{y}$, the PDWs of Fig. $\underline{2}$ also represent a temporal switching among two domains of oppositely rotating circular polarizations in the fiber, in full agreement with our subsequent numerical results that are presented in Section $\underline{4}$.

The separatrices connecting maxima of $V$ located in opposite quadrants (e.g., $\left(u_{M, 1}, v_{M, 1}\right)$ with $\left.\left(u_{M, 3}, v_{M, 3}\right)\right)$ correspond to vector dark solitons (not shown here). On the other hand, by keeping the same boundary conditions as in the example of Fig. 2, that is, searching with the shooting method for a solution linking the maximum $\left(u_{M, 3}, v_{M, 3}\right)$ with $\left(u_{M, 2}, v_{M, 2}\right)$, but using the different initial condition $\left(u_{\text {in }}(t), v_{\text {in }}(t)\right)=$ $\left(u_{M, 4}, v_{M, 4}\right)$ (that is, imposing as the initial guess for the two polarization components the values corresponding to the maximum of $V$ in the fourth quadrant), one obtains an evolution corresponding to a dark soliton in the $u(t)$ component, coupled to a bright soliton on a continuous background for the $v(t)$ component (see Fig. 3).

As can be seen by comparing Fig. $\underline{2}$ with Fig. $\underline{3}$, there are two distinct solitary-wave solutions that appear to asymptotically (i.e., for large times $t \rightarrow \pm \infty$ ) connect the same points in the phase space (i.e., adjacent maxima of the potential $V$ ). In a conservative dynamical system, there cannot be two distinct trajectories passing through the same point in the phase space. Nevertheless, the previous result may be explained by noting that Eq. (4) represent a nonintegrable, hence chaotic, dynamical system. Proper separatrix trajectories, such as those described by the polarization-domain wall of Fig. 2 , and the coupled dark and bright (on a background) solitary-wave solution of Fig. 3, may originate from two distinct, yet infinitesimally close, points in phase space, and ultimately result in widely separated trajectories (i.e., solitary-wave profiles). Thus, the nonintegrability of Eq. (4) is inherently associated with the coexistence of different types of vector solitary
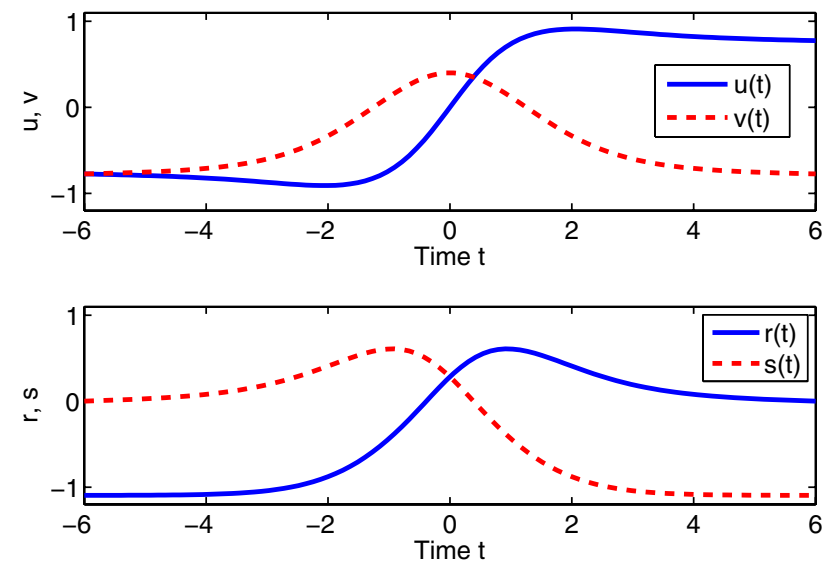

Fig. 3. (Color online) Evolution with time $t$ of the $(u, v)$ or $(r, s)$ components of a coupled dark and bright on a background vector solitary wave in a birefringent fiber.

waves, leading to the richness of solutions of the original propagation Eq. (1), depending on the specific boundary or initial conditions. As we shall see in the next section, both coupled dark and bright polarization solitons on a background as well as polarization-domain walls are indeed shown to be generated in a fiber ring laser operating in the average normal dispersion regime, depending on the specific arrangement of the intracavity polarization controller. Note that $k_{x}=5 \gamma P / 6$, where $P$ is the average power of the circular polarization domains. From Fig. 2 , one may estimate a temporal duration of the domain wall $\Delta t \simeq 4$, which is in real units $\Delta T=\Delta t\left(6 \bar{\beta}_{2} / 5 \gamma P\right)^{1 / 2}$, where $\overline{\beta_{2}}$ is the average cavity GVD.

\section{EXPERIMENTAL OBSERVATIONS OF POLARIZATION-DOMAIN-WALL STRUCTURES IN A FIBER LASER}

\section{A. Experimental Setup}

Our fiber ring laser setup is shown on Fig. 4. The laser uses a $3 \mathrm{~m}$ long EDF as the gain medium, which makes it operate at a wavelength $\lambda \approx 1.55 \mu \mathrm{m}$. The EDF is pumped at the wavelength of $1.48 \mu \mathrm{m}$ by a Raman fiber laser source delivering up to $5 \mathrm{~W}$, coupled through a 1480/1550 multiplexer WDM. Two additional multiplexers are spliced after the EDF in order to reject the remaining pumping light from the rest of the cavity, with a suppression ratio better than $30 \mathrm{~dB}$. Unidirectional laser emission is ensured by the presence of two polarizationinsensitive optical isolators surrounding the amplifier section.

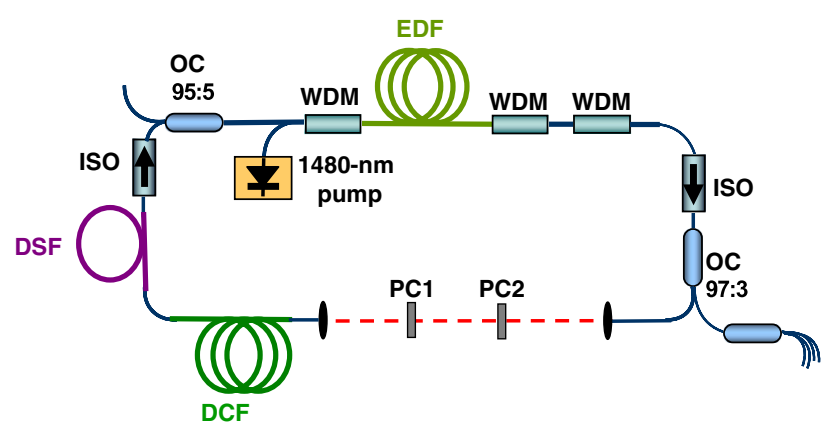

Fig. 4. (Color online) Fiber laser experimental setup. ISO, polarization-insensitive optical isolator; OC, output coupler; EDF, erbium-doped fiber; DSF, dispersion-shifted fiber; DCF, dispersioncompensating fiber; PC, quarter- and half-wave plates; and WDM, wavelength division multiplexing. 
Output couplers are placed before (95/5) and after (97/3) the EDF. The cavity comprises an open-air section, where the polarization of the intracavity light field can be modified by the insertion and orientation of quarter-wave and half-wave plates. This section is also reminiscent of a typical modelocked fiber laser setup readily obtained by sandwiching a polarizer by such sets of waveplates. However, in the present situation, no polarizer was inserted, and we measured, by injecting a tunable cw light source into the cavity and by fully varying its state of polarization (SOP), a level of polarizationdependent loss (PDL) lower than $0.2 \mathrm{~dB}$ over $10 \mathrm{~nm}$ of bandwidth. We also measured an upper boundary for the average cavity birefringence. To do so, we injected a polarized amplified spontaneous emission (ASE) signal into the cavity, while preventing the laser operation by blocking the beam in the open-air section, and monitored the output spectrum after passing through a polarizer. We found the average birefringence to be smaller than $4.10^{-6}$, or equivalently, the average beat length to be larger than $0.3 \mathrm{~m}$. This shows that in the fiber cavity, winded up fibers, spliced sections, and pigtailed components do not increase much the birefringence level, which remains consistent with the expected value $L_{b}$ for optical fibers (typically, $L_{b, \mathrm{SMF}} \sim 5 \mathrm{~m}, L_{b, \mathrm{EDF}} \sim 0.5 \mathrm{~m}$ ).

The EDF has an anomalous GVD, with a value $D=+15 \mathrm{ps} . \mathrm{nm}^{-1} \cdot \mathrm{km}^{-1}$. By inserting various lengths of a dispersion-compensating fiber $\left(D=-91 \mathrm{ps} . \mathrm{nm}^{-1} \cdot \mathrm{km}^{-1}\right)$, we are able to change stepwise the value of the path-averaged dispersion, and access to the normal dispersion regime. Fiber components are pigtailed with short lengths of SMF-28 $\left(D=+17 \mathrm{ps} . \mathrm{nm}^{-1} \cdot \mathrm{km}^{-1}\right)$. The cavity also includes a $1.5 \mathrm{~m}$ long DSF $\left(D=-2.5\right.$ ps.nm $\left.{ }^{-1} \cdot \mathrm{km}^{-1}\right)$.

The laser signal is monitored using one port of a $1: 4$ splitter spliced at the $3 \%$ output of the $97 / 3$ coupler. Then, it passes through a combination of quarter- and half-wave plates before it is sent to a polarization beam splitter, which separates the two linear orthogonal polarization components. These polarization components are finally sent to fast photodiodes whose electrical signals are recorded by a $6 \mathrm{GHz}, 40 \mathrm{GSa} / \mathrm{s}$ real-time oscilloscope (LeCroy WavePro 760Zi). Another output port of the 1:4 splitter is sent to an optical spectrum analyzer, which has a resolution of $65 \mathrm{pm}$.

Note that another EDF ring laser was previously used in [2] to analyze the fast intracavity polarization dynamics. However, this pioneering study overlooked the role of chromatic dispersion.

\section{B. Intracavity Polarization Dynamics}

We looked for vector pulse dynamics in the normal pathaveraged dispersion regime. We found coupled darkbright solitary-wave dynamics for various lengths of the dispersion-compensating fiber (1.61, 2.42, and $3.33 \mathrm{~m}$ ). Dark-bright solitary-wave dynamics, and various other collective vectorial pulse dynamics, abounded within a large domain of waveplate settings in the case of a cumulated normal dispersion $\int \beta_{2}=+0.0064 \mathrm{ps}^{2}$, obtained with the $2.42 \mathrm{~m}$ length of DCF. So, the following experimental results are obtained with this value of chromatic dispersion. In a first step, we monitor the total output intensity as the pumping power is increased. The laser threshold is obtained at less than $200 \mathrm{~mW}$ of pump power. The laser then operates in a noisy, quasi-cw regime, with the noise intensity increasing with the pump power. At a pumping level of $1 \mathrm{~W}$, the noise background drastically increases. Then, by adjusting the orientation of the waveplates PC1,2, a relatively weak self-pulsing effect can be noticed among the large noise level, with a periodicity of $73 \mathrm{~ns}$, which corresponds to the cavity repetition frequency of $13.7 \mathrm{MHz}$. Such observation is presented in Fig. $\underline{5}$.

To search for intracavity polarization dynamics, we decompose the laser signal into its orthogonal polarization components. These components are coupled and, according to the settings of the intracavity waveplates, exhibit very different dynamics. In order to exacerbate the vectorial behavior of the observed dynamics against the strong noisy background, the laser output signal is slightly averaged (five frames per averaging). Figure 6 displays the shortest vector-pulse structure found, where a coupled dark-bright solitary wave was circulating at the cavity round-trip frequency. Indeed, we can see that one polarization component displays a bright peak sitting on a very weak background, whereas at the same time, the orthogonal polarization component displays a dip on a important background. Remarkably, both pulses manifest with a significant contrast, as opposed to the faint pulsation of the total intensity. Therefore, these solitary-wave dynamics are inherently of vectorial nature.

The two temporal traces shown in Figs. $\underline{6(\mathrm{a})}$ and $\underline{6(\mathrm{~b})}$ are recorded with a rise time of $80 \mathrm{ps}$. The duration of the bright structure is $\sim 3 \mathrm{~ns}$ and that of the dark structure is $\sim 4 \mathrm{~ns}$. The nanosecond duration of the dark pulse indicates that it is not a single dark soliton, whose expected duration would be in the range of tens of picoseconds or shorter. Let us also recall that, due to the limited temporal resolution of the detection, the observation of true dark solitons in the range of $10 \mathrm{ps}$ or shorter would produce a dip of little contrast on the noisy cw background, so that it would probably remain undetected [12]. Therefore, the vector dark and bright pulses observed here do not represent the combination of a single dark soliton and a single bright soliton. However, the observed vector structure is compatible with a succession of the polarization-domain walls predicted in Section 2. Optical spectra of both bright and dark pulse components of the vector structure are presented in Fig. 6(c). The central wavelength of the dark pulse is $1556.5 \mathrm{~nm}$, and its FWHM is $120 \mathrm{pm}$. The spectrum of the

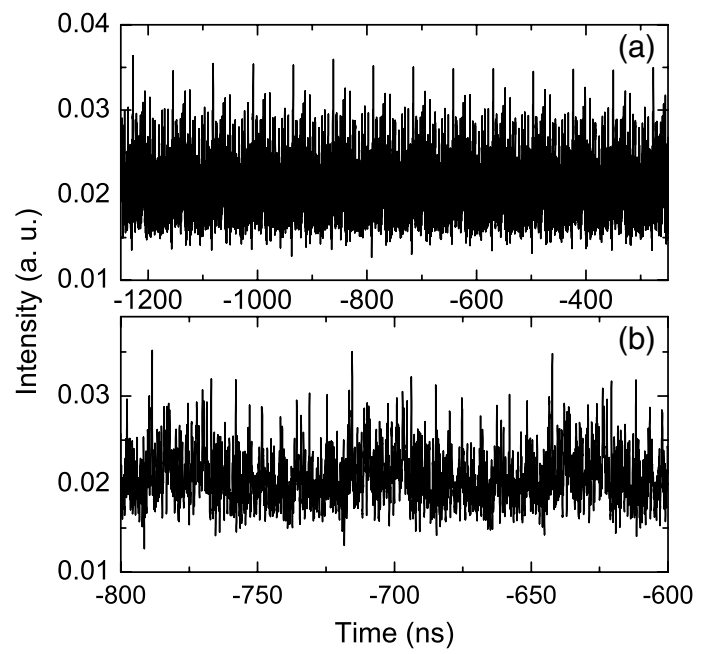

Fig. 5. Total intensity of the laser output at a pumping power of $1 \mathrm{~W}$ (a), zoomed in (b). The periodicity is $72 \mathrm{~ns}$ and corresponds to the laser-cavity round-trip time. 

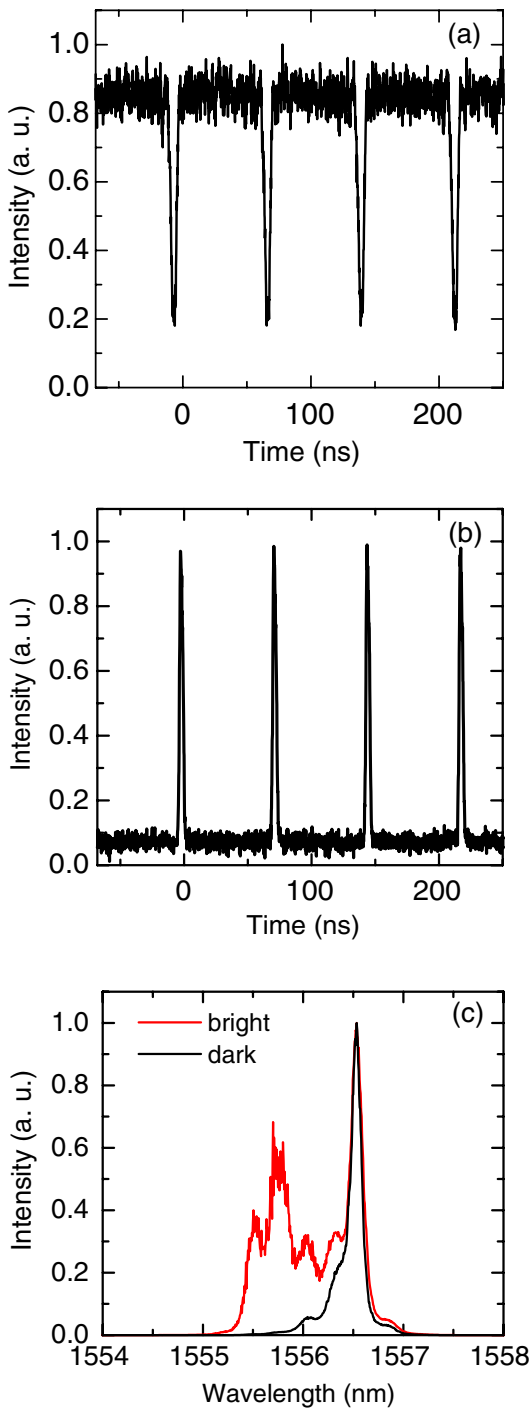

Fig. 6. (Color online) Typical dark-bright regime in the normal dispersion regime: temporal traces of the two orthogonal polarization states (a), (b) and optical spectra (c). The red-curve spectrum (left) corresponds to trace (b) and the black-curve spectrum (right) to trace (a).

bright pulse is a little more structured; its central wavelength is $1556.0 \mathrm{~nm}$ and its width is broader (FWHM = $220 \mathrm{pm})$. The difference $\Delta \lambda=0.5 \mathrm{~nm}$ between both spectral centers of mass can be understood as resulting from $\mathrm{GV}$ matching between the two polarization components, in order for the vector-localized structure to propagate one round trip after another without suffering pulse breakup. The GV matching condition $\Delta \lambda=\left(\lambda_{0}^{2} \delta /\left(2 \pi c \bar{\beta}_{2}\right)\right.$ is indeed consistent with the given value of the chromatic dispersion and that of the estimated total cavity birefringence corresponding to a retardation of the order of $10 \lambda_{0}$.

Whereas polarization-domain walls are necessarily involved in the experiment, the reality could be more complex if the vector-pulse structure would contain an unresolved internal structure, such as a bunching of subpulses. Notwithstanding, the good contrast ratio $(>70 \%)$ of the dark pulse component advocates here for plain pulses, instead of composite pulses that would readily reduce the observed contrast. In the following section, we have investigated the important issue of composite vector-pulse structures.
C. Influence of Polarization Control Inside of the Cavity The shape of vector pulsations depends essentially on the orientation of the waveplates $\mathrm{PC} 1,2$. Once a pumping power threshold, typically of the order of $1 \mathrm{~W}$, is reached, we can observe various shapes of vector solitary wave dynamics by adjusting quasi-continuously the waveplates. For instance, let us play with the orientation of the half-wave plate PC1. We first obtain the regime presented in Fig. 6 , characterized by the shortest (3-4 ns) PDW-like vector structure. From this point, other PDW-like vector structures are obtained when the angle of PC1 is varied, within a full range of $50^{\circ}$. Figure 7 presents the temporal traces of both orthogonal polarization states for various values of the PC1 angle and their corresponding optical spectra. Here, we have recorded in real time the orthogonal states of polarization without any averaging. This reveals that the vector-pulse structure is composed of not just one, but a series of chaotic PDW-like transitions that travel the cavity. We indeed notice the presence of several spikes well above the average noise level, first of all in the bright pulse component part. This is a clear indication of a composite structure involving different time scales, which is only partly resolved temporally.

The width of the PDW-like vector structure presented in Figs. $7(\mathrm{a})$ and $7(\mathrm{~b})$ is $8 \mathrm{~ns}$. The duration increases to 12 and $20 \mathrm{~ns}$, while developing a squared overall shape, in Figs. 7(d) and 7(e) and Figs. 7(g) and 7(h), respectively. Corresponding optical spectra, for both polarization components, appear in Figs. 7(c), 7(f), and 7(i). Optical spectra hardly change over the whole range of waveplate orientations considered here (from $26^{\circ}$ to $36^{\circ}$ ). Remarkably, the difference between the centers of the spectra remains equal to $0.5 \mathrm{~nm}$, in agreement with the GV matching argument developed above. By adjusting the orientation of PC1, we not only change the duration but also the details of the composite vector structure. We note that a short and relatively intense peak appears during the back transition of the dark pulse component, in Figs. 7(d) and 7(g). The temporal duration of the intense peak is around $280 \mathrm{ps}$. This peculiar substructure could be related to the existence of a dark-bright flash as predicted recently [13]. We have also recorded optical autocorrelation traces, using background-free second-harmonic generation. These traces, whose examples are displayed in Fig. $\underline{8}$, are difficult to obtain due to the large noise level and the relatively low peak intensity. However, they all display a small coherence peak of the order of $5 \mathrm{ps}$, which is compatible with the predicted time scale of the PDW transitions.

Increasing further the orientation of PC1 leads to the breakup of the localized square waves into a set of vector multipulse solitary waves that fill the entire cavity. This corroborates further the hypothesis of composite PDW-like structures. Interestingly, the two spectra of orthogonal polarization components become almost identical, which indicates that the GV matching condition can be relaxed in the delocalized pulse regime.

\section{NUMERICAL SIMULATIONS USING A DETAILED MODEL}

In order to verify, by detailed numerical simulations, the spontaneous generation of PDWs in the fiber laser, and retrieve some of the complex features highlighted above, we used 

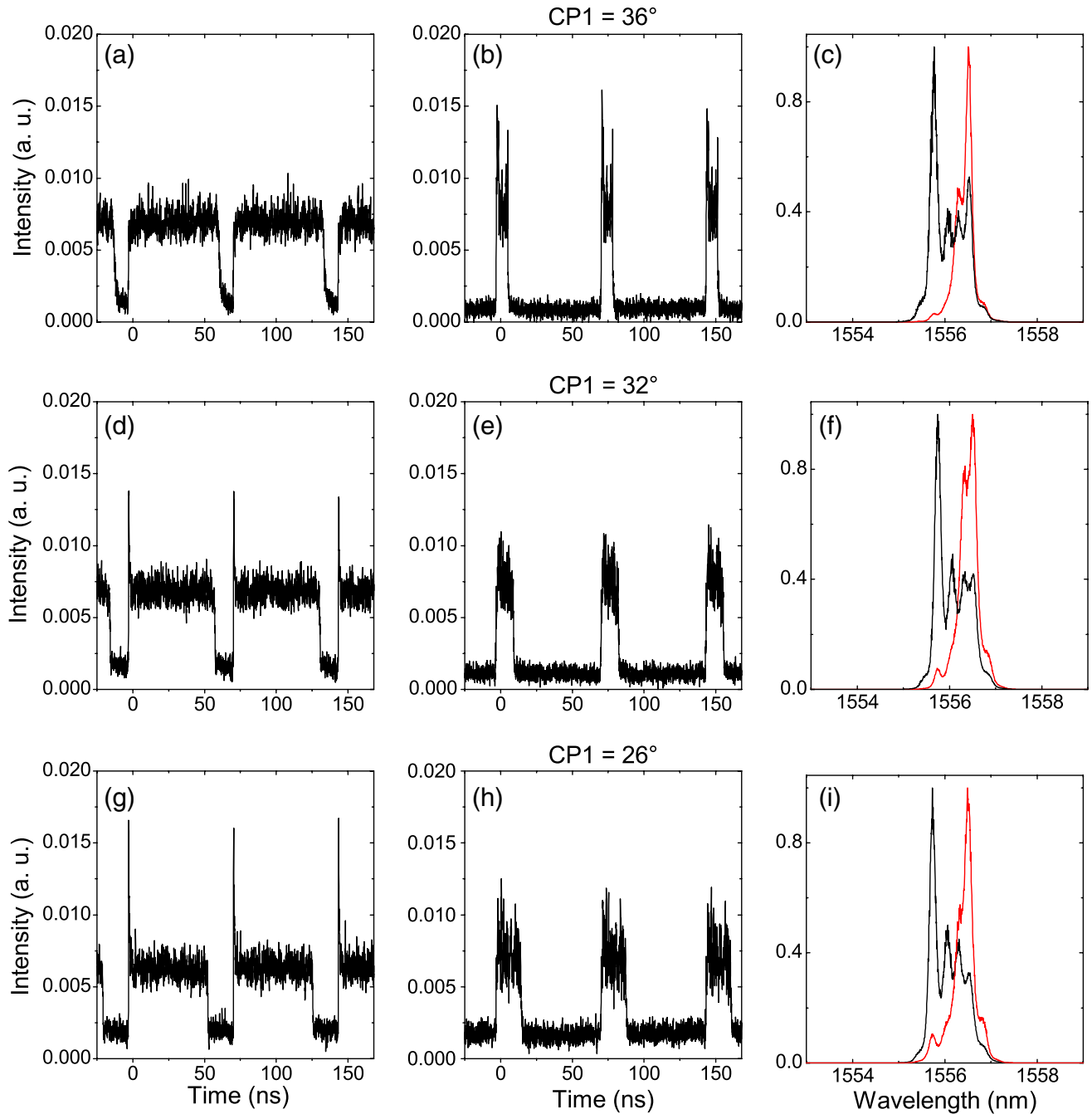

Fig. 7. (Color online) Influence of polarization control: temporal traces of the two orthogonal polarization states and corresponding optical spectra for three different orientations of CP1. Dark PDW-like structures are characterized by red-curve spectra (right), and bright PDW-like structures are characterized by black-curve spectra (left).

the commercial VPITransmissionMaker and VPIComponentMaker software to compute the buildup of polarization structures from ASE noise that is recirculated through the various components (erbium doped fiber amplifier (EDFA), isolators, fibers, and couplers) of the experimental setup for the case of an average normal GVD. We injected in the EDFA $1.6 \mathrm{~W}$ of pump power at $1480 \mathrm{~nm}$. The EDFA was modeled on the basis of the bidirectional propagation equations for signals and the two-level rate equations for ion populations. The emission and absorption spectra were specified in terms of cross sections. The erbium lifetime was set to $10 \mathrm{~ms}$, the erbium concentration was $2.4 \times 10^{25} \mathrm{~m}^{-3}$, and the erbium core radius and overlap factors were $3.1 \mu \mathrm{m}$ and 0.81 , respectively. The EDFA model includes self-phase modulation due to the Kerr nonlinearity, and is resolved in both the longitudinal and the transverse directions.

For the sake of simplicity, we neglect in the simulation the presence of the small residual PDL and the nonzero fiber birefringence-induced GV walk-off among the two polarization components of the fibers. However, we verified that the observed results are robust with respect to the introduction of the measured small PDL and GV mismatch. Figure 9 shows the numerically computed optical spectrum as extracted from the $97: 3$ output coupler after the first pass through the EDFA, which reproduces the frequency distribution of the ASE spectral intensity. The black arrow indicates the residual $1480 \mathrm{~nm}$
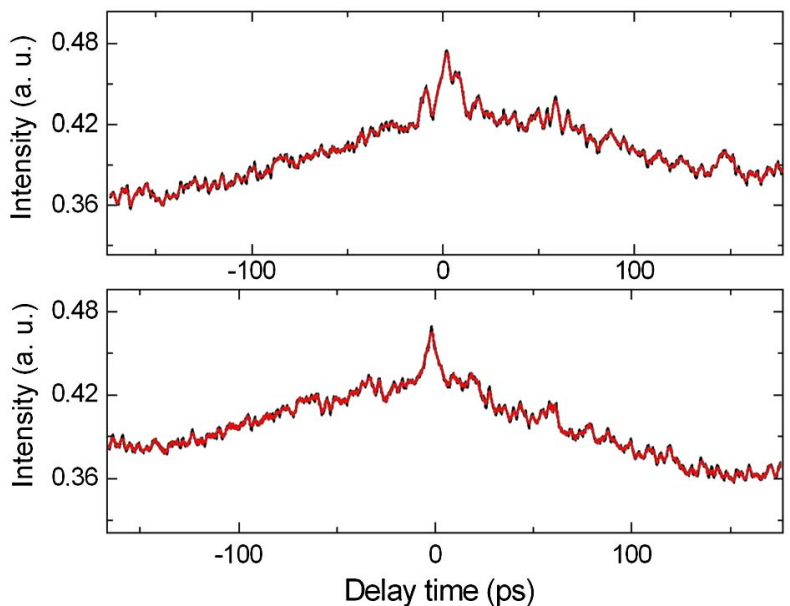

Fig. 8. (Color online) Two examples of recorded background-free autocorrelation traces, showing small coherence peaks shorter than $10 \mathrm{ps}$. 
pump power. Figure $\underline{9}$ also shows the random SOP of the initial ASE noise: here we display the $S_{3}=\left(I_{R}-I_{L}\right) / I_{T}$ component of the normalized Stokes vector $\left(I_{T}=I_{R}+I_{L}\right)$, which measures the relative intensity difference between right and left circular SOPs.

After $N=1900$ round trips through the laser, the optical field has substantially evolved. Figure 10 shows the computed output optical spectrum; the spectrum peak has moved to $1560 \mathrm{~nm}$, and its shape is approximately triangular, with about $2 \mathrm{~nm}$ full width at half maximum. Moreover, Fig. 10 shows that the intracavity SOP is now spontaneously organized in a train of randomly spaced PDWs, which represent a complex of abrupt temporal transitions between the two orthogonal circular polarization states. On the other hand, the total intracavity intensity exhibits irregular temporal fluctuations with a typical time scale of a few picoseconds and the average value of $P=5 \mathrm{~W}$. For the average cavity dispersion $\overline{\beta_{2}}=$ $+4.5 \mathrm{ps}^{2} / \mathrm{km}$ and nonlinear coefficient $\gamma=1.9 \mathrm{~W}^{-1} \mathrm{~km}^{-1}$, the analytical prediction of Section $\underline{2}$ yields a typical PDW duration of $3 \mathrm{ps}$, in good quantitative agreement with the numerical results of Fig. 10 .

\section{DISCUSSION AND CONCLUSIONS}

The observation of vector pulsations in an EDF ring cavity is readily accessible, yet the observations indicate versatile dynamics, whose physical origin has remained so far controversial. With this background, we have proposed a novel theoretical model predicting the formation of polarizationdomain walls in the realistic environment of a fiber ring laser, namely taking into account the typical orders of magnitudes of the physical effects at play in the fiber cavity. Hence, we have found that the dominant effects are merely, in the absence of significant PDL, the optical Kerr effect and the chromatic dispersion. Despite the simplicity of the related model, it

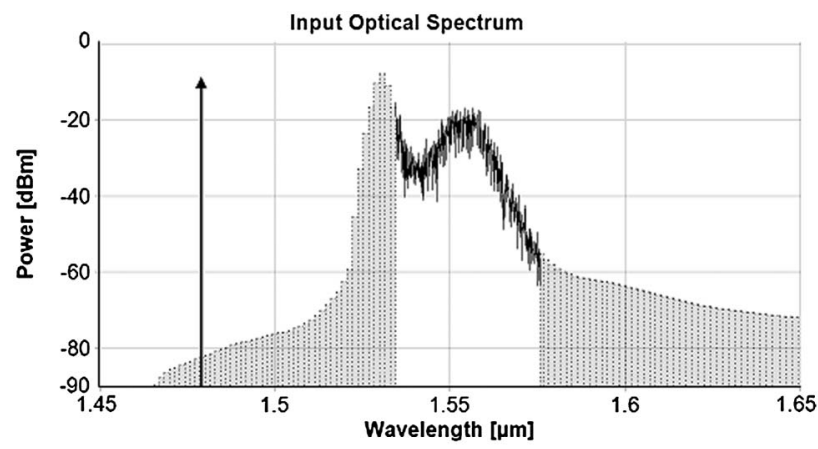

S3

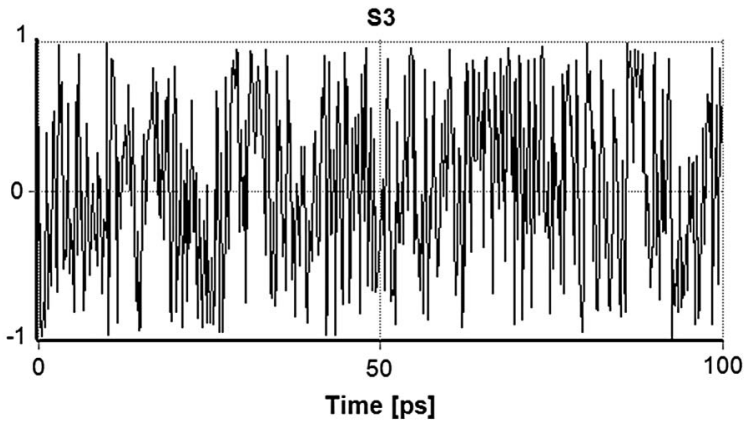

Fig. 9. At the first cavity round trip: numerically simulated optical spectrum and time evolution of the SOP $\left(S_{3}\right.$ parameter) at the exit of the EDFA.

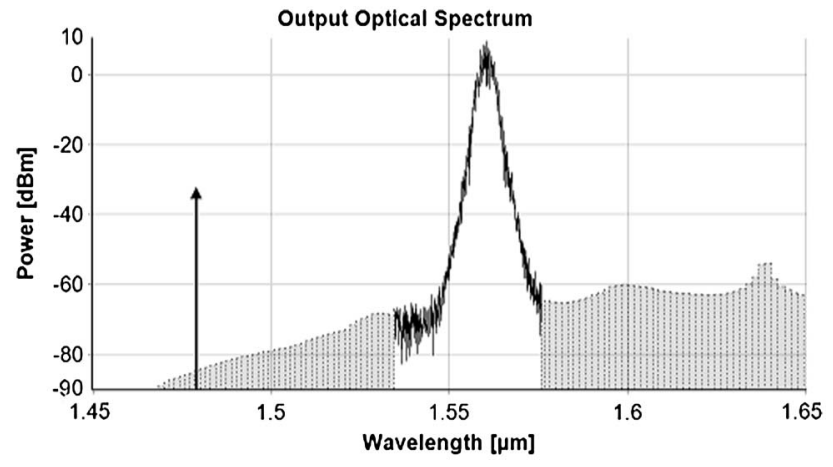

S3

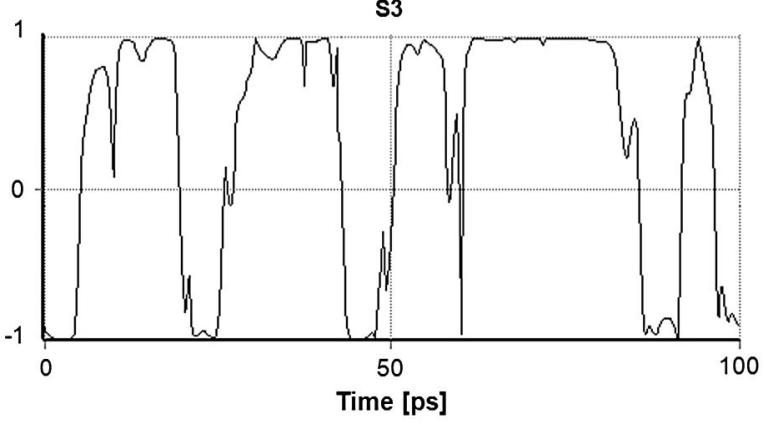

Fig. 10. As in Fig. 3, after $N=1900$ circulations in the laser.

allowed us to find several solitary waves. In particular, we have highlighted two types of PDW transitions, connecting the same initial and final orthogonal states of polarization. The first one is the "classic" PDW, which corresponds to a constant total intensity. It is similar to the one found in [8], but importantly, the condition for ultralow birefringence is relaxed in our situation. The second PDW transition is characterized by a more pronounced transition shape, where the total intensity is no longer constant. Hence, we have identified it as a vector dark and bright solitary wave. The prediction of several PDW-like transitions is an important step to address the complexity of the experimental observations of selfpulsing polarization vector dynamics. We have clarified the scope of these observations by stating that only the outcome of long-lived transitions can be observed, since the temporal resolution of the detection chain, of the order of $100 \mathrm{ps}$, is much larger than the predicted PDW transition, around 4 ps. It has turned out that the experimental observation indicates the existence of composite PDW-like transitions, which are only partly resolved using our $6 \mathrm{GHz}$ detection electronics, but are, however, highlighted for some particular settings of the intracavity waveplates, in the case of long square-shaped vector pulses, as well as consecutive to the composite pulse breakup filling the entire cavity. In order to bridge the gap between the model and the experiment, since in the latter, the influence of background noise seems essential to trigger the various vector pulsations, we have started detailed numerical simulations. These simulations confirm the spontaneous formation of a series of PDW transitions arranged in a chaotic manner, as a result of the noise influence.

\section{ACKNOWLEDGEMENTS}

C. L. and Ph. G. acknowledge support from the Agence Nationale de la Recherche ("Solicristal" project ANR-2010-BLANC0417-01). The work of S. W. was carried out with support from 
the Conseil Regional de Bourgogne, the iXCore Foundation, and the Italian Ministry of University and Research (MIUR) through grant contract 2008MPSSN.

\section{REFERENCES}

1. P. Grelu and N. Akhmediev, "Dissipative solitons for modelocked lasers," Nat. Photonics 6, 84-92 (2012).

2. Q. L. Williams, J. Garcia-Ojalvo, and R. Roy, "Fast intracavity polarization dynamics of an erbium-doped fiber ring laser: inclusion of stochastic effects," Phys. Rev. A 55, 2376-2386 (1997).

3. G. D. Van Wiggeren and R. Roy, "High-speed fiber-optic polarization analyzer: measurements of the polarization dynamics of an erbium-doped fiber ring laser," Opt. Commun. 164, 107-120 (1999).

4. H. D. I. Abarbanel, M. B. Kennel, M. Buhl, and C. T. Lewis, "Chaotic dynamics in erbium-doped fiber ring lasers," Phys. Rev. A 60, 2360-2374 (1999).

5. H. Zhang, D. Y. Tang, L. M. Zhao, and X. Wu, "Observation of polarization domain wall solitons in weakly birefringent cavity fiber lasers," Phys. Rev. B 80, 052302 (2009).

6. H. Zhang, D. Y. Tang, L. M. Zhao, and R. J. Knize, "Vector dark domain wall solitons in a fiber ring laser," Opt. Express 18, 4428-4433 (2010).
7. H. Y. Wang, W. C. Xu, W. J. Cao, L. Y. Wang, and J. L. Dong, "Experimental observation of bright-dark pulse emitting in an all-fiber ring cavity laser," Laser Phys. 22, 282-285 (2012).

8. M. Haelterman and A. P. Sheppard, "Polarization domain walls in diffractive or dispersive Kerr media," Opt. Lett. 19, 96-98 (1994).

9. M. Haelterman and A. P. Sheppard, "Bifurcations of the dark soliton and polarization domain walls in nonlinear dispersive media," Phys. Rev. E 49, 4512-4518 (1994).

10. F. Gutty, S. Pitois, P. Grelu, G. Millot, M. D. Thomson, and J. M. Dudley, "Generation and characterization of $0.6 \mathrm{THz}$ polarization domain-wall trains in an ultralow-birefringence spun fiber," Opt. Lett. 24, 1389-1391 (1999).

11. B. Crosignani, S. Piazzolla, P. Spano, and P. Di Porto, "Direct measurement of the nonlinear phase shift between the orthogonally polarized states of a single-mode fiber," Opt. Lett. 10, 89-91 (1985).

12. S. Coen and T. Sylvestre, "Comment on dark pulse emission of a fiber laser,” Phys. Rev. A 82, 047801 (2010).

13. F. Baronio, A. Degasperis, M. Conforti, and S. Wabnitz "Solutions of the vector nonlinear Schrödinger equations: evidence for deterministic rogue waves," Phys. Rev. Lett. 109, 044102 (2012). 